\title{
Factors influencing young women's contraceptive choices
}

Lily Claringbold, Lena Sanci, Meredith Temple-Smith

\section{Background and objectives \\ Australia's high rate of unintended pregnancy could be lowered by increasing long-acting reversible contraception (LARC) use. Influences on Australian women's choice of less effective methods are not well understood. This qualitative study explored factors that influence young women's contraceptive choices.}

\section{Methods}

Semi-structured interviews were conducted with women aged 18-24 years until data saturation. Interviews were audio-recorded, transcribed and thematically analysed.

\section{Results}

Contraceptive choice was influenced by factors including contraceptive knowledge, side effects, cultural norms, fear, control and social context. Women often initiated contraceptive use for non-contraceptive purposes such as management of acne or period pain, and failed to reconsider new methods when needing contraception, revealing one possible explanation for low LARC use.

\section{Discussion}

Findings indicate that women's contraceptive choices need regular review. Information about relative efficacy of contraceptive methods is necessary should women's reasons for using contraception include birth control in addition to non-contraceptive benefits.
DESPITE VARYING contraceptive options, unintended pregnancy in Australia is a public health concern. ${ }^{1}$ Approximately half of all Australian women have one unintended pregnancy in their lifetime. ${ }^{2}$ One new cross-sectional Australian study found that within the past 10 years, $26 \%$ of pregnancies were unintended, and $30 \%$ of these ended in abortions. ${ }^{3}$

Long-acting reversible contraception (LARC) includes three main methods: copper and progestogen intrauterine devices (IUDs), administered once every 10 and five years respectively; progestogen subdermal implants, administered every three years; and progestogen injections, administered three-monthly. Implants and IUDs are over $99 \%$ effective at preventing pregnancy, while progestogen injections are $94-99.8 \%$ effective depending on the user. ${ }^{5}$ IUDs are recommended by organisations such as the Royal Australian and New Zealand College of Obstetricians and Gynaecologists (RANZCOG) as first-line contraception, regardless of pregnancy history. ${ }^{4}$ However, despite its advantages, LARC is not commonly chosen by young Australian women. ${ }^{6}$

Approximately $70 \%$ of Australian women of reproductive age employ some form of birth control. ${ }^{1}$ On the basis of 2013 data, $27-34 \%$ of women used oral contraception, 20-23\% used condoms and $13-21 \%$ used vasectomy/tubal ligation. LARC is the least popular method, with only $1-4 \%$ of women choosing an implant, and $1-3 \%$ using IUDs. ${ }^{6}$
Young women aged 18-24 years often choose less reliable contraception such as oral contraception and condoms. ${ }^{6}$ At the time of first unintended pregnancy for women aged 18-23 years, 39\% were using oral contraceptives, $29 \%$ were using condoms and $19 \%$ were using withdrawal. ${ }^{7}$ One study showed that young women aged 18-24 years have the highest rate of general practitioner (GP) consultation for contraception and unplanned pregnancies when compared with other age groups. ${ }^{8}$ While their desire to not become pregnant can be implied by use of contraception, the rate of unintended pregnancies suggests incorrect or inconsistent contraceptive practices. Influences on young women's contraceptive choices include poor knowledge, negative side effects and cultural norms..$^{9-11}$ One US study found that with comprehensive contraceptive counselling, over $70 \%$ of young women chose the more reliable LARC over oral contraception, ${ }^{12}$ although contraception was provided free of charge in this study. The initial cost of LARC is likely to be an influence on choice, even though it is more cost effective than other contraceptive choices.

Research on young Australian women's contraceptive choices is limited and mainly survey based. This qualitative study aimed to explore factors influencing Australian young women's contraceptive choices to inform GP education. 


\section{Methods}

This study used a qualitative descriptive approach to explore factors influencing young women's contraceptive choices. Qualitative description is a study design suitable for research 'aiming to gain firsthand knowledge of patients', relatives' or professionals' experiences with a particular topic'. ${ }^{13}$

Convenience sampling was used to recruit women aged $18-25$ years from the University of Melbourne, Parkville campus for individual semi-structured interviews at a convenient time and place. Participants were recruited using flyers, online advertising on the student portal and snowball sampling. No reimbursement was offered. Interviews proceeded until data saturation was reached and no new themes emerged. The study was approved by the University of Melbourne Health Sciences Human Ethics Sub-Committee (project ID 1749418).

Interviews were conducted in person or by Skype, audio-recorded and transcribed verbatim. Participants signed a consent form and completed a short demographic questionnaire prior to questions commencing. The interview schedule was informed by literature and expert advice of clinicians and researchers and was pilot-tested. It used a series of prompts to elicit general contraceptive knowledge, decision to start using contraception, contraception education and choice of contraceptive method (Box 1).

Transcripts were de-identified to ensure participant anonymity, and data management was assisted by NVivo software. Content analysis was used to thematically analyse data using both inductive and deductive approaches. ${ }^{14}$ A coding framework used interview prompts, codes identified from published literature and codes identified from transcript data. Codes were categorised into themes to reflect key concepts. Consensus on themes was reached by the study team.

\section{Results}

Twenty interviews were conducted with women aged 18-24 years; 19 face to face and one via Skype. Interviews were approximately 30-45 minutes in duration, with an average of 20 minutes discussing detailed interview questions.

\section{Demographics}

Table 1 shows participant demographics. Most participants lived in share housing and were not in a relationship. Half the participants identified as Australian, and half were international students: four from Western countries and six from Asian countries.

\section{Initial choice of contraception}

Table 2 presents information about initial contraceptive choice. Just over half of the participants stated that women commenced contraception for reasons other than preventing pregnancy alone. Half made their decision about the type of contraception they wanted before a consultation with a doctor - a decision that may have been made with little knowledge. When asked to list contraceptive methods they knew of,

\section{Box 1. Interview schedule}

\section{Knowledge about contraception}

1. What comes to mind when I say 'contraception'?

2. Do you talk to your friends about contraception?

3. What types of contraception do you know about?

-What do you know about these methods?

- How effective do you think they are?

\section{Decision to use contraception}

4. Why do you think young women start using contraception?

- Why do you think men start using contraception?

\section{Knowledge about contraceptive options}

5. Can you tell me what you know or what you've heard about the oral contraceptive pill?

- Where have you learnt about it from?

- Why would/wouldn't you use it?

6. Have you heard about long-acting reversible contraception? From where?

- Do you have any idea what that would be?

7. Can you tell me what you know or what you've heard about the Implanon?

- Where have you heard about it from?

- Have you discussed it with your family/mother? What do they think about it?

- Why would/wouldn't you use it?

8. What do you know about intrauterine devices?

- What have you heard about it and from where?

- Have you discussed it with your family/mother? What do they think about it?

- Why would/wouldn't you use it?

\section{Learning about contraception}

9. You mentioned learning about contraception through ...

10. How did/do you learn about contraception?

- Parents, friends, school, doctors, the internet, partners?

- Does this change over time?

- How reliable do you think these sources are?

\section{Choosing a method of contraception}

11. What factors are most important when choosing a method of contraception?

- Why would you choose one method over another?

- Why do women change contraceptive method?

- Would the type of relationship you're in influence the choice?

- Who/what has the greatest impact on your choice?

\section{Debrief}


most could list only four methods, most commonly the oral contraceptive pill and condoms.

\section{Summary of results}

Factors influencing women's views on contraception were condensed into six main themes: social context, contraceptive knowledge, side effects, cultural norms,

\section{Table 1. Participant demographics ( $n=20)$}

\begin{tabular}{ll}
\hline Age (years) & 7 \\
$18-20$ & 6 \\
$21-22$ & 7 \\
$23-24$ & 7
\end{tabular}

\begin{tabular}{lr}
\hline Nationality & 10 \\
Australian & 2 \\
UK & 2 \\
Chinese & 2 \\
Malaysian & 1 \\
US & 1 \\
French & 1 \\
South Korean & 1 \\
Thai & 15 \\
\hline Relationship status & 5 \\
Single & 15 \\
In a relationship & 4 \\
\hline Living arrangement & 1 \\
Share house & \\
Family home & \\
Residential college & \\
\hline
\end{tabular}

Table 2. Initial choice of contraception $(n=20)$

n

\section{Why do women start using} contraception?

Birth control alone

Non-contraceptive positive side effects alone

Combination of both

When did you decide on what contraception to use?

Before consultation with physician

After consultation with physician

Not using contraception control and fear. Those within a woman's social sphere were often the source of contraceptive information, and included family, friends, the internet, school and interactions with GPs. Lack of contraceptive knowledge, negative side effects of contraception and cultural norms relating to contraceptive use have been well documented in previous literature as influences on women's contraceptive choices. ${ }^{9-11}$ Only the new and less frequently reported themes of fear, control and positive side effects are presented in this paper, alongside aspects of social context.

\section{Social context \\ Family}

Many women discussed contraception with family but expressed that this did not influence their choices. Some conveyed they had a mutual 'don't ask, don't tell' arrangement, while others expressed that their family's culture or religion influenced their choices, largely due to fear of discovery.

She was like 'Yeah, as long as you've done your research and you know, like, what it's about and that kind of thing then that's your choice'. (Participant [P] 12)

She said, 'Oh, do we need to have the awkward contraception talk?' And then I said, 'I'm on the pill' and left the room. (P4)

You hide those things away from your parents. (P20)

\section{Friends}

Experiences of women's friends often influenced choices; however, most expressed that discussing contraception with friends was simply a way to share experiences, gain knowledge and solicit support.

No one's going to talk about their birth control unless something bad happens. (P1)

If I have a friend who I trust who's raved about a certain one, I'll be much more inclined to get it or consider it. (P11)

I trust my friends [...] it's better to hear from someone that you know and trust, so I'd say they're pretty credible. (P8)
Internet

The internet was regularly used to gain knowledge of options or to better understand potential side effects. Some women drew directly from reliable resources; however, most sought information from forums or blogs.

There's one called Bedsider, which has like a really good array of information on contraception. (P17)

I think a lot more people speak more freely about it and it's less uncomfortable, because it's usernames and stuff, so people are very forward about stuff to do with reproductive health and sexual health. (P3)

It's a way to feel informed in a situation where, despite like $50 \%$ of the population being female ... it's still such a clouded topic. (P5)

\section{School education}

Women who did not receive any education about contraception generally were international students or attended a very religious school. Most did receive some school education about contraception, although all except one deemed it insufficient or unmemorable. Often, the oral contraceptive pill and condoms were the only options outlined, or LARC was not discussed as a realistic option.

I went to a religious school. They didn't expect you to have sex until you got married ... there was no need to talk about intimacy, sexual relations, you know, any of that kind of safe sex. (P17)

It was so uneventful or not well described that I do not recall. (P2)

I think it was very much pitched as 'the two options of contraception are the pill and condoms.' (P4)

Interactions with health professionals Interactions with health professionals varied. Some women indicated that their doctors did not influence their contraceptive choices. 
When I went to get the pill I said, 'I want the pill', and when I went to get the Implanon I said, 'I want the Implanon.' (P4)

Many perceived the oral contraceptive pill to be the only option discussed, or felt that their doctor persuaded them towards or away from LARC, often conflicting with their preferences.

You go to the doctor and say, 'I'm having sex', and they straight away just prescribe it to you, without too much explanation or thought into it. (P11)

He said that IUD, for me, would be very painful ... he really discouraged me from it. (P16)

I wanted to stop using the Implanon; the doctor tried to convince me to stay on it a bit longer. (P4)

Others shared positive experiences with doctors.

She listened to my concerns about stuff and then we both decided that the IUD was the best option so yeah, that was great. (P6)

Fear

The theme of fear had three foci: adverse side effects of contraception, invasiveness of LARC and stigma surrounding use of contraception.

The perception of stigma surrounding contraception use strongly influenced women's contraceptive choices, particularly those from non-Western countries. Social stigma also prevented some from obtaining contraceptive knowledge.

I think it's easier not to use condoms because, like, then where would I keep them? And I don't want to be seen buying them as well. (P20)

I don't think people use it as much because of ... society's stigma. The fact that you're going into a pharmacy and getting one, like that - that experience in itself wouldcould intimidate you. (P8)

Two women were concerned with the potential for copper IUDs to rust.
One pregnant lady I was working with said that it actually rusted inside of her. (P17)

My biology teacher told us a story where ... she didn't bother to take it out and it rusted. (P20)

Fear surrounding potential negative effects of hormones concerned many and was often the reason women avoided the oral contraceptive pill.

I'm just so touchy about any sort of hormone control. It just freaks me out. (P6)

I was a bit scared because I used to have depression and I didn't want to have mood swings because of taking the pill. (P15)

Women were discouraged from LARC use because of perceived potential pain of insertion of IUDs.

It sounded like it was really painful ... (P3)

... I don't really want to deal with, you

know, severe pain if I can avoid it. (P16)

While issues of invasiveness arose for IUD, this was particularly perceived for the implant, which was seen as unnatural or disturbing by some.

I also know you can still feel it once it's in and that really grosses me out. (P6)

It's kind of creepy to be honest. (P20)

\section{Control}

The theme of control featured frequently in participants' responses, not only in controlling pregnancy but also in regard to menstruation and contraception.

Participants from Australia and the US expressed a desire for a contraceptive method they felt they could control. Their negative views of LARC were based on the inability to insert or remove it themselves.

It's really wanting to find a way to have ... agency over your own body. (P4)

It's in my arm; it's still in my arm. I can't get it out. (P1)
Some participants' contraceptive choices were based on the capacity to control menstruation.

I was hoping to ... start getting my life under control, my periods under control. (P13)

Women expressed general distrust in male partners to use contraception appropriately, and so contraceptive choices were often influenced by the desire to be in control of their risk of unintended pregnancy.

I mean, you can't always trust a guy to ... do everything correctly. (P6)

Most guys I've had sex with don't like wearing condoms, so I needed to ... take matters into my own hands. (P13)

\section{Positive side effects}

An unexpected theme that arose was the positive side effects of the contraceptive pill, which for many women may outweigh the benefits of LARC. Three subthemes emerged: acne management, heavy or painful period management and control of the menstrual cycle.

The most common positive side effect influencing women's contraceptive choices was acne management.

I was using it for my skin. (P20)

I had friends in high school who were taking contraception pill, not because of contraception, but because of acne. (P8)

Another positive side effect was management of heavy or painful periods.

They use it as a method just to control their periods if they were far too painful or heavy or very irregular. (P11)

Finally, some chose oral contraception as they simply wanted to control their menstruation for convenience.

I wanted to skip it because ... busy times, and I always have it during my exams, which really affects my performance. (P13)

Often, this was the primary reason young women initiated contraceptive use, and 
they continued to use oral contraception due to its convenience and familiarity.

I was using it for my skin, and something else-to give regular periods, and then when I had a boyfriend I just kept using it, as obviously it was already a contraception in a way ... I didn't have to say anything to anyone. (P20)

\section{Discussion}

This small qualitative study aimed to address the lack of literature around the influences on young women's contraceptive choices through 20 semi-structured interviews with women aged 18-24 years. Questions about initial contraceptive choices revealed that many started using a contraceptive method for reasons other than pregnancy prevention alone, and that the decision about what contraceptive method to use was made prior to consultation with a doctor. Several themes in the data confirmed previous reports in the literature such as a lack of contraceptive knowledge, negative side effects of contraception and cultural norms. ${ }^{9-11}$ However, new perspectives offered by this research include the influence of positive side effects of oral contraception, and themes of fear and control. These were often experienced differently by the individual depending on her circumstances; family; information sources such as friends, the internet and school; and interactions with GPs.

Misinformation about certain methods of contraception, particularly IUDs, was quite common among participants, and this is especially concerning given the participants were all undertaking tertiary education and might be expected to bring some level of enquiry to their contraceptive choices. While it is evident from the literature that misconceptions among both the public and practitioners remain about the safety and suitability of IUDs, little is known about the experiences of pain on insertion in this group, as few studies examining the pain of IUD insertion include nulliparous women. ${ }^{15}$

The finding that most women did not initiate their contraceptive methods for contraceptive purposes alone has not previously been reported. Eleven of 20 women initiated contraceptive use for either just the positive side effects of the oral contraceptive pill, or a combination of the positive effects and contraceptive purposes. Although side effects have been frequently reported to affect women's contraceptive choices, they almost exclusively refer to negative side effects; ${ }^{9-11}$ this may contribute to the continuing high rate of oral contraception use, and consequent low uptake of LARC. Similarly, most women had decided what method of contraception to use before consultation with their GPs and used their GPs as a 'contraception shop' to acquire it. This is problematic because GPs may assume that women requesting a specific method of contraception are well informed, yet our research suggests that this is often not the reality. On the basis of our findings, it is suggested that GPs advise women about other appropriate contraceptive methods, not just the one being requested, to ensure they are fully informed.

Our findings about contraceptive information sources for young women confirm those reported internationally ${ }^{16-18}$ and include discussions with friends, school education and searching the internet. Despite consulting friends or the internet most often for contraceptive advice, most women recognised their limited reliability. Likewise, although many women reported unsatisfactory interactions with GPs, they considered GPs a reliable information source, providing women with the opportunity to ensure they are fully informed about contraceptive options and to revisit these options regularly. If women start using the oral contraceptive pill for non-contraceptive purposes such as acne control or painful menstrual cycles, our results indicate that these women may continue to use it, regardless of whether their circumstances have changed to include protection against unwanted pregnancy. Without GPs prompting this discussion, young women may not be aware of vital details regarding the use of this method for contraception, such as information about missing a pill, interactions with other medications, or emergency contraception. Likewise, they are unlikely to consider the benefits of alternative and more efficacious options such as LARC.

\section{Strengths and limitations}

Potential recruitment bias is a limitation of this study, as women interested in contraception may have been likely to volunteer. Likewise, participants were recruited through a single university. Further research should examine views of those outside higher education, and younger adolescent women. A strength of this study was the qualitative methodology, which allowed detailed exploration of factors that influence young women's contraception choices. The student researcher was also a young woman of similar age. Participants appeared comfortable to openly discuss their views of contraception with a peer, thereby allowing unique insight. However, because of the sensitive nature of the issue and ethics constraints, participants were advised at the start of interviews that they were not required to disclose details of their own contraceptive history but may instead choose to refer to 'a friend's' situation instead of their own to ensure privacy. Therefore, although participants appeared to be clearly describing their own personal experiences, this may not be the case for all. The number of women interviewed was sufficient for data saturation.

\section{Conclusions}

This study used qualitative research to explore influences on young women's contraceptive choices. Participants were volunteer local and international students in a tertiary education setting. Several key themes confirmed previous literature; however, a new research finding may contribute to the low LARC uptake - namely that many women initiate a contraceptive method for non-contraceptive purposes, and often decide this before consultation with a GP. As many young women are not fully informed about all contraceptive options, changing circumstances in young women's lives offer the opportunity to reconsider the most appropriate contraceptive 
method. Discussions with women about available contraceptive methods, including relative efficacy in preventing pregnancy, may increase LARC uptake for some and help lower the rate of unintended pregnancies in Australia.

\section{Implications for general practice}

- Young women may have limited knowledge of contraceptive options and therefore require comprehensive contraceptive counselling.

- As young women's circumstances and reasons for contraceptive use are likely to change over time, a woman's choice of contraception needs to be revisited to ensure it is appropriate and to maximise efficacy.

\section{Authors}

Lily Claringbold BSc (Hons), MPhil student, Department of General Practice, University of Melbourne, Parkville, Vic. lily.claringbold@unimelb. edu.au

Lena Sanci MBBS, PhD, FRACGP, Head of Department, Department of General Practice, University of Melbourne, Parkville, Vic Meredith Temple-Smith BSc, MPH, DHSc, Deputy Head of Department and Director of Research Training, Department of General Practice, University of Melbourne, Parkville, Vic

Competing interests: None.

Funding: None.

Provenance and peer review: Not commissioned, externally peer reviewed.

\section{References}

1. Richters J, Grulich AE, Visser RO, Smith AMA, Rissel CE. Sex in Australia: Contraceptive practices among a representative sample of women. Aust N Z J Public Health 2003;27(2):210-16. doi: 10.1111/j.1467-842X.2003.tb00810.x.

2. Marie Stopes International. Real choices: Women, contraception and unplanned pregnancy. Melbourne: Marie Stopes Australia, 2008.

3. Taft AJ, Shankar M, Black KI, Mazza D, Hussainy S, Lucke JC. Unintended and unwanted pregnancy in Australia: A cross-sectional, national random telephone survey of prevalence and outcomes. Med J Aust 2018;209(9):407-08. doi: 10.5694/mja17.01094.

4. Royal Australian and New Zealand College of Obstretrics and Gynaecologists. Long acting reversible contraception (LARC). East Melbourne, Vic: RANCGOG, 2014

5. Trussell J. Contraceptive failure in the United States. Contraception 2011;83(5):397-404. doi: 10.1016/j.contraception.2011.01.021.

6. Family Planning NSW. Reproductive and sexual health: An Australian clinical practice handbook. 2nd edn. Ashfield, NSW: Family Planning NSW, 2011.
7. Coombe J, Harris ML, Wigginton B, Lucke JC, Loxton D. Contraceptive use at the time of unintended pregnancy: Findings from the Contraceptive Use, Pregnancy Intention and Decisions study. Aust Fam Physician 2016;45(11):842-48.

8. Mazza D, Harrison C, Taft A, et al. Current contraceptive management in Australian general practice: An analysis of BEACH data. Med J Aust 2012;197(2):110-14. doi: 10.5694/mja11.11599.

9. Dixon SC, Herbert DL, Loxton D, Lucke JC. 'As many options as there are, there are just not enough for me': Contraceptive use and barriers to access among Australian women. Eur J Contracept Reprod Health Care 2014;19(5):340-51. doi: 10.3109/13625187.2014.919380.

10. Garrett CC, Keogh LA, Kavanagh A, Tomnay J, Hocking JS. Understanding the low uptake of long-acting reversible contraception by young women in Australia: A qualitative study. BMC Womens Health 2015;15:72. doi: 10.1186/s12905015-0227-9.

11. Mills A, Barclay L. None of them were satisfactory: Women's experiences with contraception. Health Care Women Int 2006;27(5):379-98. doi: 10.1080/07399330600629468.

12. Secura GM, Allsworth JE, Madden T, Mullersman JL, Peipert JF. The Contraceptive CHOICE Project: Reducing barriers to longacting reversible contraception. Am J Obstet Gynecol 2010;203(2):115.e1-7. doi: 10.1016/j. ajog.2010.04.017.

13. Neergaard MA, Olesen F, Andersen RS, Sondergaard J. Qualitative description - The poor cousin of health research? BMC Med Res Methodol 2009;9:52. doi: 10.1186/1471-2288-9-52.

14. Miles MB, Huberman AM. Qualitative data analysis: An expanded sourcebook. 2nd edn. Thousand Oaks, CA: SAGE, 1994.

15. Lyus R, Lohr P, Prager S. Use of the Mirena LNG-IUS and Paragard CUT380A intrauterine devices in nulliparous women. Contraception 2010;81(5):367-71. doi: 10.1016/j. contraception.2010.01.010.

16. Sundstrom B. Fifty years on 'the pill': A qualitative analysis of nondaily contraceptive options. Contraception 2012;86(1):4-11. doi: 10.1016/j. contraception.2011.10.016.

17. Vogt $C$, Schaefer M. Disparities in knowledge and interest about benefits and risks of combined oral contraceptives. Eur J Contracept Reprod Health Care 2011;16(3):183-93. doi: 10.3109/13625187.2011.561938.

18. Whittaker PG, Merkh RD, Henry-Moss D, Hock-Long L. Withdrawal attitudes and experiences: A qualitative perspective among young urban adults. Perspect Sex Reprod Health 2010;42(2):102-09. doi: 10.1363/4210210. 